\title{
The Role of Motion Extrapolation in Amphibian Prey Capture
}

\author{
Bart G. Borghuis ${ }^{1,2}$ and Anthony Leonardo ${ }^{1}$ \\ ${ }^{1}$ Janelia Research Campus, Howard Hughes Medical Institute, Ashburn, Virginia 20147, and 2Department of Anatomical Sciences and Neurobiology, \\ University of Louisville, School of Medicine, Louisville, Kentucky 40202
}

Sensorimotor delays decouple behaviors from the events that drive them. The brain compensates for these delays with predictive mechanisms, but the efficacy and timescale over which these mechanisms operate remain poorly understood. Here, we assess how prediction is used to compensate for prey movement that occurs during visuomotor processing. We obtained high-speed video records of freely moving, tongue-projecting salamanders catching walking prey, emulating natural foraging conditions. We found that tongue projections were preceded by a rapid head turn lasting $\sim 130 \mathrm{~ms}$. This motor lag, combined with the $\sim 100$ ms phototransduction delay at photopic light levels, gave a $\sim 230 \mathrm{~ms}$ visuomotor response delay during which prey typically moved approximately one body length. Tongue projections, however, did not significantly lag prey position but were highly accurate instead. Angular errors in tongue projection accuracy were consistent with a linear extrapolation model that predicted prey position at the time of tongue contact using the average prey motion during a $\sim 175 \mathrm{~ms}$ period one visual latency before the head movement. The model explained successful strikes where the tongue hit the fly, and unsuccessful strikes where the fly turned and the tongue hit a phantom location consistent with the fly's earlier trajectory. The model parameters, obtained from the data, agree with the temporal integration and latency of retinal responses proposed to contribute to motion extrapolation. These results show that the salamander predicts future prey position and that prediction significantly improves prey capture success over a broad range of prey speeds and light levels.

Key words: delays; extrapolation; motion; prediction; prey capture; retina

\section{Significance Statement}

Neural processing delays cause actions to lag behind the events that elicit them. To cope with these delays, the brain predicts what will happen in the future. While neural circuits in the retina and beyond have been suggested to participate in such predictions, few behaviors have been explored sufficiently to constrain circuit function. Here we show that salamanders aim their tongues by using extrapolation to estimate future prey position, thereby compensating for internal delays from both visual and motor processing. Predictions made just before a prey turn resulted in the tongue being projected to a position consistent with the prey's pre-turn trajectory. These results define the computations and operating regimen for neural circuits that predict target motion.

\section{Introduction}

The neuronal chain of action that links sensory input to motor output introduces significant delays between behavioral responses and the events that evoke them. Sensory transduction

Received Aug. 25, 2015; revised 0ct. 5, 2015; accepted 0ct. 15, 2015.

Author contributions: A.L. and B.G.B. designed research; A.L. and B.G.B. performed research; A.L. and B.G.B. analyzed data; A.L. and B.G.B. wrote the paper.

This work was supported by the Howard Hughes Medical Institute. We thank Bill Mowrey, John Tuthill, and Vivek Jayaraman for helpful comments on the manuscript; the Janelia Fly Core facility for providing the fruit flies used in this study; and the Janelia Vivarium for help maintaining the salamander colony.

The authors declare no competing financial interests.

Correspondence should be addressed to either of the following: Dr. Bart G. Borghuis, Department of Anatomical Sciences and Neurobiology, University of Louisville, School of Medicine, Louisville, KY 40202, E-mail: bart.borghuis@louisville.edu; or Dr. Anthony Leonardo, Janelia Research Campus, Howard Hughes Medical Institute, 19700 Helix Drive, Ashburn, VA 20147, E-mail: leonardoa@janelia.hhmi.org.

DOI:10.1523/JNEUROSCI.3189-15.2015

Copyright $\odot 2015$ the authors $\quad 0270-6474 / 15 / 3515430-12 \$ 15.00 / 0$ and signal processing in the visual system require tens to hundreds of milliseconds (Aho et al., 1993; Leonardo and Meister, 2013), and additional delays are incurred by the computations needed for motor control (Franklin and Wolpert, 2011), and for contracting muscles to produce force (Deban and Dicke, 1999). For behaviors such as picking up a stationary object these delays are inconsequential, but most behaviors are inundated with selfmotion and object motion. Here, the duration of sensorimotor delays relative to the timescale of motion in the world becomes crucial. The challenge of swatting a fly illustrates this principle: executing a guided reach takes hundreds of milliseconds (Fisk and Goodale, 1988), comparable with the average inter-turn interval of a flying fly (Tammero and Dickinson, 2002; Mronz and Lehmann, 2008; Muijres et al., 2014). If the hand is directed to the fly's location at the time of detection, when the hand arrives, the fly is likely to be gone. 
Nervous systems have evolved at least two approaches for ameliorating delays in sensorimotor control. The first are direct optimizations to minimize the delay. Classic examples are the large axon, short sensory-motor loops characterizing high-speed behaviors, such as escape responses (von Reyn et al., 2014) and flight control (Rowell and Pearson, 1983) in invertebrates, and the myelination of axons to increase action potential transmission speed in both vertebrates and invertebrates (Hartline and Colman, 2007). These biophysical improvements are effective but limited by physical law: the response delay of a sensory neuron cannot be zero and residual delays, although brief, may still exceed the requirements for effective behavior. This constraint has led to a second approach to sensorimotor control, which uses prediction to surpass the fundamental limit set by physical delays. Indeed, various organisms use models of the motor apparatus to plan movements (Kawato, 1999; Mehta and Schaal, 2002; Card and Dickinson, 2008b) and to time them to predicted future events (Wolpert et al., 1995; Mischiati et al., 2015). For example, in humans, prediction is used to extrapolate the trajectories of moving objects (Land and McLeod, 2000; Zago et al., 2009; Etchells et al., 2010; de Rugy et al., 2012) and may compensate for sensory delays (Nijhawan, 1994; Brenner and Smeets, 2000; Nijhawan, 2002). Animal models of these processes, where the underlying neural control mechanisms are accessible, remain scarce.

We explored the role of prediction in sensorimotor behavior using amphibian prey capture as a model system in which the interaction between visual, motor, and target properties can be explored quantitatively and linked with neuronal circuit dynamics (Aho et al., 1993; Dicke and Roth, 1994; Monroy and Nishikawa, 2009). Salamanders use high-speed tongue projections to catch moving prey. Typical sensory delays in the retina are $\sim 100 \mathrm{~ms}$ in bright light (Aho et al., 1993), and tongue projection generally requires $<10 \mathrm{~ms}$ (Deban and Dicke, 1999). However, additional kinematic delays from head movements are likely involved (Roth, 1987). Whether the overall visuomotor delay requires compensation via predictive control depends on the delay magnitude and the statistics of prey motion leading up to the time of capture, which are not known. Here, we filmed freely moving salamanders and fruit fly prey under a variety of conditions and quantified tongue projection accuracy and its dependence on prey motion statistics and visuomotor delays. We found that salamander tongue projections were aimed at a predicted future prey position that was well described by a linear extrapolation model. Motion extrapolation explained high prey capture accuracy across a wide range of prey velocities and light levels. Parameters of the extrapolation model were well matched to the visuomotor delays and broadly consistent with the properties of the retinal ganglion cells proposed to contribute to such processes (Berry et al., 1999; Leonardo and Meister, 2013; Johnston and Lagnado, 2015).

\section{Materials and Methods}

All experiments were conducted according to protocols approved by the Institutional Animal Care and Use and Institutional Biosafety Committees of the Howard Hughes Medical Institute, Janelia Research Campus. Wild-caught salamanders (Eurycea guttolineata) of unidentified sex were acquired from a commercial vendor (Glades Herp). No differences in individual performance were noted.

The video recording setup is shown schematically in Figure $1 A$. A salamander and a fruit fly (Drosophila melanogaster; referred to as "prey" throughout the text) were paired in a behavior arena of $15 \times 15 \times 4 \mathrm{~cm}$, positioned under a high-speed video camera (Photron SA1.1). An infrared (IR) illumination panel (peak wavelength $850 \mathrm{~nm}$; Advanced Illumi- nation) placed below the arena's IR-transmitting floor permitted highcontrast imaging at a wavelength outside the visible range of the salamander (Perry and McNaughton, 1991). A transparent acrylic lid prevented the salamander from leaving the arena without affecting the quality of the video image. Two diffuse white LED light sources (Advanced Illumination) placed directly adjacent to the camera were used to set the ambient light level in the arena ( $>4.5$ log unit range; $0.01-250$ $\mu \mathrm{W} / \mathrm{cm}^{2}$ ), approximating the range of illuminations in which amphibians are known to hunt. Amphibians can capture prey at even dimmer light levels, but under these conditions response rates are reduced (Roth, 1987). Behavior was recorded at 4000 frames per second with a $1 / 4000-$ $1 / 10,000$ shutter speed; image resolution was $0.14 \mathrm{~mm}$ per pixel. Salamanders were adapted to each light level for at least 15 min before prey presentation. Elapsed time between the introduction of a prey into the behavior arena and the time of capture ranged from $10 \mathrm{~s}$ to $2 \mathrm{~min}$; salamanders typically ate $15-20$ prey before cessation of the capture behavior.

Prey capture was constrained to the horizontal plane by using a wingless Drosophila mutant $\left(n u b^{-/-}\right.$; Fly Core, Janelia Research Campus, Ashburn, Virginia) that did not fly but showed wild-type walking behavior (typical walking speed $\sim 17 \mathrm{~mm} / \mathrm{s}$ ). Prey was prevented from climbing the arena walls using one of two methods. In approximately half of the recordings, a silicon suspension (Sigma Cote; Sigma-Aldrich) was applied to the walls. In all other recordings, a temperature-controlled heat strip (Kapton; Southeast Thermal Systems) lined the arena wall. Surface temperature of the heat strip was feedback controlled and set to $30^{\circ} \mathrm{C}$ to effectively repel the prey. Meshcovered air vents prevented excess heat build-up in the arena. In the presence of Sigma Cote, some flies still attempted to climb the wall; projections during these trials were discarded.

Data analysis. Data are presented as mean \pm SD unless noted otherwise. Errors are reported as root-mean-square (RMS) where applicable.

Video records of salamander and prey behavior leading up to a tongue projection (2-5 s duration; 8000-20,000 frames) were analyzed off-line, using custom algorithms (MATLAB, The MathWorks; code available upon request; see Fig. $1 B$ ). Coordinates of the prey, salamander eyes, and the direction of the tongue were defined as the centers of an ellipse (prey), circles (salamander eyes), and Gaussian (tongue cross-section) fitted to the respective objects in each frame of the recorded prey capture attempt (least-squares fit, MATLAB; resolution $<0.14 \mathrm{~mm}$ ). A line normal to the line connecting the centers of the eyes defined the salamander's head orientation. Salamander head turns were detected by applying an amplitude and angular velocity threshold criterion to the head orientation over time (50 ms running boxcar average; thresholds: $5^{\circ}$ amplitude, $10 \%$ velocity). We distinguished orienting head turns, which were not followed by a tongue projection, and projection head turns, which were immediately followed by a tongue projection ( $<15$ ms delay).

Tongue projections were accompanied by a retraction of the eyes into the eye sockets, which decreased the distance between the salamander's eyes in a highly stereotyped manner (see Fig. $1 C$ ). This marker for tongue projections, apparently related to flexing of the tongue projection musculature, was used in off-line analysis to automatically detect projections in each recorded image sequence. All projections (both hits and misses) were included in the analysis. Successful captures (hits) were defined by the salamander consuming the prey, as observed in the video recordings. All other projections were classified as misses.

Plethodontid salamanders do not make eye movements (MartinezConde and Macknik, 2008). Therefore, the prey's image on the retina could be computed directly from the position of the prey relative to the salamander's head orientation. Because the analysis and model simulations did not require binocular information, measurements of prey angular position, size, and speed were made relative to the "head center," defined as the point midway between the two eyes.

Tongue projection error was measured in units of azimuthal angle and depth. Azimuthal error was defined as the angle between a line from the head center to the center of mass of the prey and a line from the head center to the center of mass of the tongue pad ( $\alpha_{\text {tongue }}$; see Fig. 1B). Azimuthal error was measured in the last image frame, $0.25 \mathrm{~ms}$ before tongue contact with the prey, when the tongue line was still well defined. Center of mass of the tongue pad cross-section was resolved to $<0.1^{\circ}$. 
Depth accuracy was defined as the Euclidian distance between the prey and the tip of the tongue at the time of maximum extension. For the purpose of locating tongue and prey, the time interval between the tongue just before contact with the prey and the tongue at maximum extension was negligible. Vertical offset between the salamander's head and the prey's body was estimated to be $<5 \mathrm{~mm}$. Because the behavior was constrained to the $X-Y$ plane, elevational projection errors were not considered in the analysis.

We calculated for each projection a continuity index (see Fig. $2 B, C$ ). The continuity index was computed as the RMS error between a line fit to the X-Y coordinates of the prey position before the head turn and the X-Y coordinates of the prey after the onset of the head turn (or after the tongue projection if no head turn was detected). As such, the continuity index expressed the average departure of the prey's trajectory after the onset of the head turn from the linear extension of its trajectory before the head turn. Continuity indices ranged from 0.003 to $3.87 \mathrm{~mm}$ (mean $0.35 \pm 0.39 \mathrm{~mm}$ ). For comparison, the length of a fruit fly is $2.5-3.0 \mathrm{~mm}$, and the continuity index would be $2.8 \mathrm{~mm}$ if the fly made a right angle turn at its average speed of $17 \mathrm{~mm} / \mathrm{s}$ during a $120 \mathrm{~ms}$ head turn.

For the analysis presented in Figure 7, we obtained model parameters from the recorded data, grouped into two light levels (0.01-0.2, 2.0$\left.250.0 \mu \mathrm{W} / \mathrm{cm}^{2} ; n=34, n=91\right)$. These values were chosen to obtain a sufficient number of projections in each group. Using all projections in each light level group, the optimal delay for the extrapolation model converged on $0 \mathrm{~ms}$ (data not shown). A zero delay is physiologically impossible and arose because the full dataset includes projections aimed at stationary flies as well as flies moving on a straight trajectory toward the salamander. In both cases, the angular velocity would be zero, and the best prediction parameters were not well constrained. We avoided this degeneracy by limiting the dataset to prey with substantial angular velocity ( $>3 \%$ s; dim, bright: $n=24, n=65)$. This restricted "moving prey" dataset gave the physiologically plausible (non-zero) delay value used in the model analysis to predict all projections, including both stationary and moving prey.

Most of the presented data show errors evaluated in angular units. We chose angular coordinates to characterize our results in terms of the prey image motion that would occur on the salamander retina. This should facilitate future studies on the neural basis of motion extrapolation, which will be grounded in the same angular coordinate system. Evaluation of errors in units of Euclidean distance between fly center and tongue-pad center gave similar results (data not shown). The data in Figure $7 C-E$ show model predictions of tongue position based on parameter values that were obtained from the recorded dataset, including the predicted data. Because (1) the dataset is large, (2) only trajectories with significant prey motion were included in the parameter fitting, and (3) tongue angular errors were near-normally distributed (see Fig. $3 C, D$ ), a jackknife method, where prediction of one tongue projection would be based on parameters obtained by averaging all-but-the-predicted projection, would give similar results.

\section{Results}

Target tracking behaviors are found in a wide variety of organisms and use many different strategies. In some cases, nonpredictive strategies suffice. For example, Blowflies (Boeddeker and Egelhaaf, 2003; Boeddeker et al., 2003) and tiger beetles (Haselsteiner et al., 2014) will chase and capture a moving target by steering toward a visually delayed image of the prey's angular position at each moment in time. Dragonflies, on the other hand, intercept prey using both prey angular position and prey angular velocity (Olberg et al., 2000; Mischiati et al., 2015). The dependence on velocity allows the interception trajectory to be predictive and directed toward the prey's future location. Human eye saccades, too, use a predictive strategy based on the target's velocity (Etchells et al., 2010). In amphibian prey capture, whether guidance of the tongue is predictive is not known.

At the moment the salamander decides to strike, it is operating on a neural image of the prey with a sensory delay of at least $\sim 100$ ms, the latency of an amphibian retinal ganglion cell responding to a change in light intensity (Leonardo and Meister, 2013). Orienting the head (Roth, 1976) and launching the tongue (Deban et al., 2007) cause additional motor delays. In this work, we considered the significance of these delays in three possible models of tongue control: an extrapolation model in which the tongue was aimed at the prey's predicted position after the full sensorimotor delay; a motor-lag model that accounted for prey movement after the visual delay but not the motor delay; and a visuomotor-lag model that aimed at a delayed prey image with no prediction at all (Fig. 1D). Assessing the effectiveness of each model required quantifying the statistics of the salamander's and prey's motion, how tongue projection accuracy varied depending on these parameters, and what part of the prey the salamander aimed at (e.g., the prey's center or leading edge; Fig. 1D).

We began by reproducing salamander prey capture under laboratory conditions. Salamanders in nature prey upon small walking invertebrates (Roth, 1987). We filmed tongue-projecting salamanders ( $n=6)$ hunting walking prey (268 events) in a small behavior arena (4000 frames/s; see Materials and Methods; Fig. $1 A)$. Computer-controlled white LED lights set the luminance in which the salamander had to detect and capture the prey, while invisible IR lighting beneath the floor of the arena ensured adequate luminance contrast to the overhead camera at all ambient light levels (0.01-250 $\left.\mu \mathrm{W} / \mathrm{cm}^{2}\right)$. We used custom off-line, frameby-frame object tracking algorithms to extract the motion trajectories of salamander's head and the prey, as well as the timing, direction, distance, and azimuthal accuracy of each tongue projection in each video (Fig. $1 B, C$; see Materials and Methods).

The first question we addressed is whether it is necessary for the salamander to predict prey motion when interacting with freely moving prey. Prior work has focused on kinematic studies of tongue projection to immobilized prey where prediction is unnecessary (Deban and Dicke, 1999), or on the accuracy during projections to repetitive linear motion of artificial prey (Aho et al., 1993). Real prey, however, exhibit complex curved motion patterns, with varying degrees of predictability. We found that prey walking speed showed a distinctly bimodal distribution: one mode of the velocity distribution represented walking (average speed $\sim 17 \mathrm{~mm} / \mathrm{s}$, range $12-24 \mathrm{~mm} / \mathrm{s}$ ), whereas the second mode represented pauses $(0 \mathrm{~mm} / \mathrm{s})$ between bouts of walking (Fig. 2A). Walking speed was largely independent of ambient light level, except at the lowest light level $\left(0.01 \mu \mathrm{W} / \mathrm{cm}^{2}\right)$, where the average speed during the walking mode was reduced by $30 \%$. Prey typically showed no avoidance response to the salamander and escape responses were rare. Indeed, for the majority of captures $(>95 \%)$, prey motion trajectories showed no change in bearing just before a tongue projection (Fig. $2 B, C$ ). Instead, the prey's typical trajectory was continuous: a line fit to the twodimensional trajectory before the tongue projection $(0.10 \pm 0.10$ $\mathrm{mm}$ RMS error) provided a highly accurate prediction of the prey's location after the tongue projection $(0.35 \pm 0.39 \mathrm{~mm}$ RMS error; see Materials and Methods). Thus, while prey speed was an important factor during prey capture, evasive behavior of the prey was not. These data indicate that linear extrapolation of the prey's motion would be a plausible strategy to cope with sensorimotor delays.

The difficulty of prey capture increases with increasing prey angular speed and distance, requiring faster reactions and higher projection accuracy, respectively. We examined the distributions of prey angular speed, angular size, and distance across all tongue projections, both successful and unsuccessful (Fig. 2D-F). These distributions represent the prey motion statistics that the salamander 
A
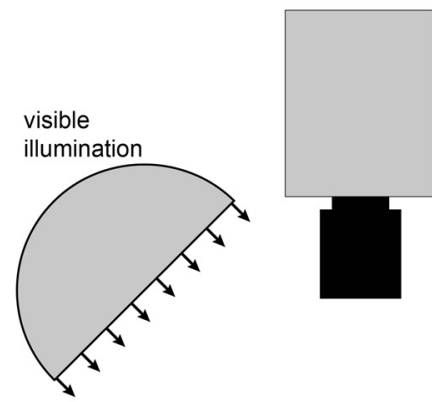

high-speed video camera $1024 \times 1024$ pixels 4000 frames/s
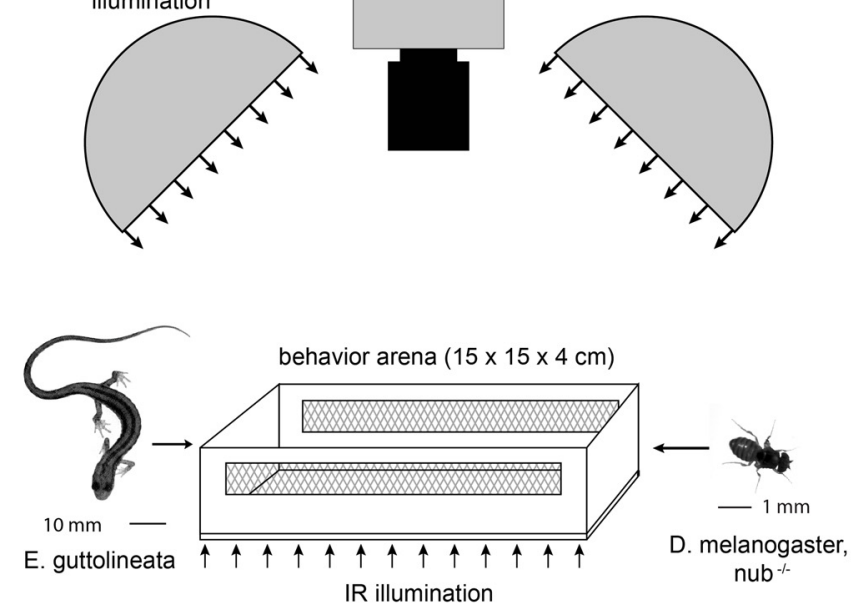

C

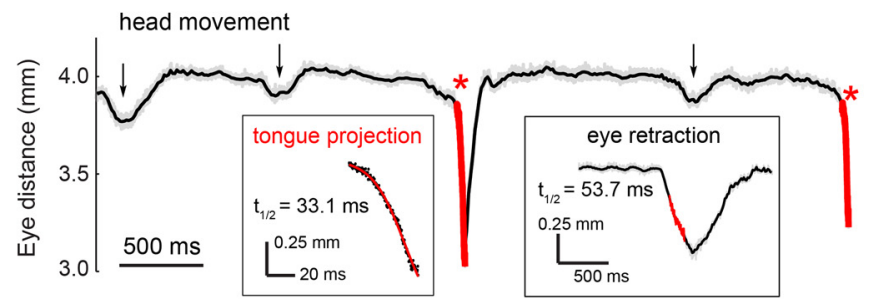

B

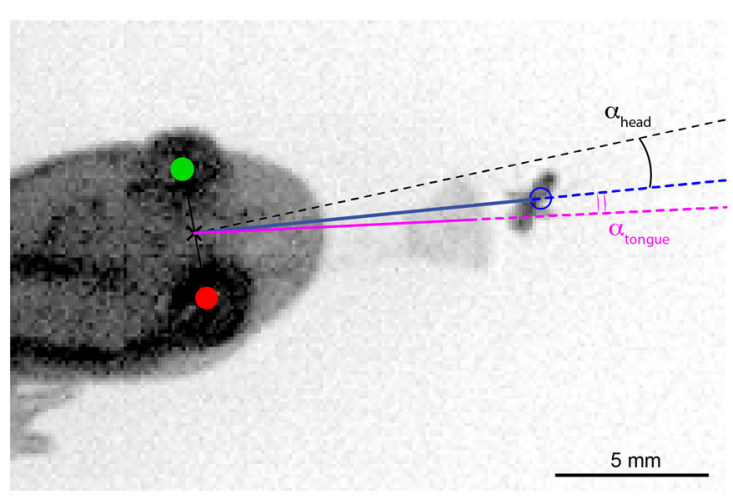

D i

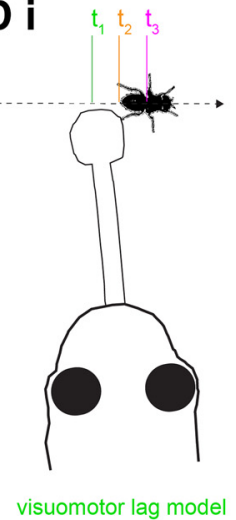

ii

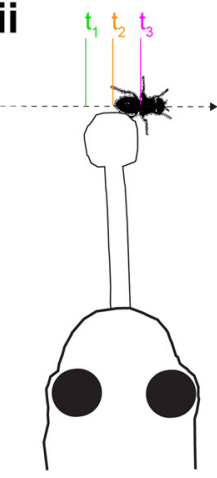

motor lag model

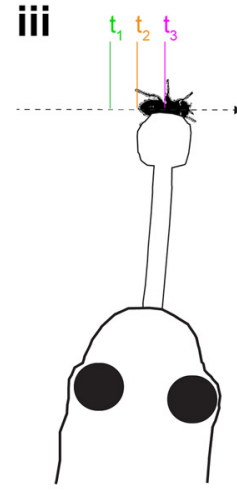

extrapolation model

Figure 1. Quantitative analysis of salamander tongue projection accuracy and prey motion. $A$, Salamander and fruit fly (prey) were paired in a behavior arena, and prey capture was filmed at 4000 frames per second. Infrared illumination under the translucent arena floor provided adequate contrast in the camera image, whereas white LED dome lights set the visible light level for the behavior. $B$, Projection accuracy was defined as the angle between the line from the reference point midway between the two eyes to the prey (blue line) and the line from the reference point to the center of the tongue pad at the end of the extended tongue (magenta line). C, Example of the salamander inter-eye distance during a single predation event. Inter-eye distance decreased rapidly at the onset of the tongue projection (asterisks). These rapid decreases were used for automated detection of all tongue projections (hits and misses) in the recorded image sequence. Changes in inter-eye distance associated with tongue projections were distinguished from those caused by other behaviors, such as head turns and eye retractions (arrows) based on their faster temporal kinetics and larger amplitude, as calculated from an exponentially decaying function fit to the data (red lines), triggered by a deviation exceeding $0.2 \mathrm{~mm}$ from the moving average intereye distance ( $25 \mathrm{~ms}$ window). D, Schematic representation of the three tongue guidance models tested in this study. At time ${ }_{2}$, the salamander detects the prey and initiates prey capture. However, due to sensorimotor delays, it sees the prey image lagged in the past, at position $\mathrm{t}_{1}$. Motor delays prevent the tongue from contacting the prey until it attains position $\mathrm{t}_{3}$. The prey moves throughout this process, and the salamander can thus aim at (i) the visuomotor-lagged position of the prey, compensating for neither delay; (ii) the motor-lagged position of the prey, compensating only for the visual delay; or (iii) the extrapolated position of the prey, compensating for both delays.

deemed viable for predation. The average angular speed was $33.7 \pm$ $44^{\circ} / \mathrm{s}$, distance $12.4 \pm 4.0 \mathrm{~mm}$, and angular size $9.0 \pm 3.9^{\circ}$ (all mean \pm SD, $n=268$; Fig. $2 D-F$ ). Thus, diverse prey conditions (i.e., slow- and fast-moving, near, and far; Fig. $2 G$ ) evoked tongue projections, indicating that the salamander's prey selection mechanism is broadly tuned. This broad range included high angular speeds and large distances, suggesting that, for projections to be accurate, predictive control is likely required (Fig. 1D).

\section{Robust projection accuracy during prey motion implies predictive control}

Prey typically moved (Fig. $2 A$ ), challenging the mechanism that aims the salamander's tongue. Despite the broad range in prey motion parameters (Fig. $2 D-G$ ), capture success was high: $91 \%$ at the optimal light level $\left(10 \mu \mathrm{W} / \mathrm{cm}^{2}, n=64\right.$; Fig. $\left.3 A\right)$, and tongue projections remained effective over a $4.5-\log _{10}$ unit range around the optimal light level $\left(66 \%\right.$ hits at $0.01 \mu \mathrm{W} / \mathrm{cm}^{2}, n=38 ; 71 \%$ hits at $\left.250 \mu \mathrm{W} / \mathrm{cm}^{2} ; n=96\right)$. To quantify projection accuracy we defined the angular error as the angle between the tongue line and the line of sight to the center of the prey, relative to the salamander's head center (Fig. 1B; for definitions, see Materials and
Methods). The magnitude of the angular errors decreased monotonically with increasing light level from $7.4 \pm 1.3^{\circ}$ at $0.01 \mu \mathrm{W} /$ $\mathrm{cm}^{2}$ to $2.7 \pm 0.3^{\circ}$ at $250 \mu \mathrm{W} / \mathrm{cm}^{2}$ (Fig. $3 B$ ). The average angular size of the prey at the time of projection remained $\sim 9.0^{\circ}$ across light levels.

To test whether the salamander compensates for prey motion, we examined how angular error varied as a function of prey angular speed and prey angular size. If tongue projections were driven by the visuomotor-lag model and were not predictive, then the tongue would lag behind the prey by one visuomotor delay (Fig. 1D). In this case, the angular error would increase proportionally with angular velocity, and the slope of this relation would reflect the visuomotor delay. We found, instead, that angular errors were relatively independent of prey angular velocity and showed no obvious trend to increasing errors with increasing speed as would be expected from a delay-sensitive tongue aiming strategy (Fig. 3C). Angular error also showed no obvious dependence on prey angular size, indicating that the salamander accurately targets the center of the prey rather than its leading edge (Figs. 1D, 3D). Because the complexity of the prey's naturalistic motion trajectories resulted in a broad distribution of 

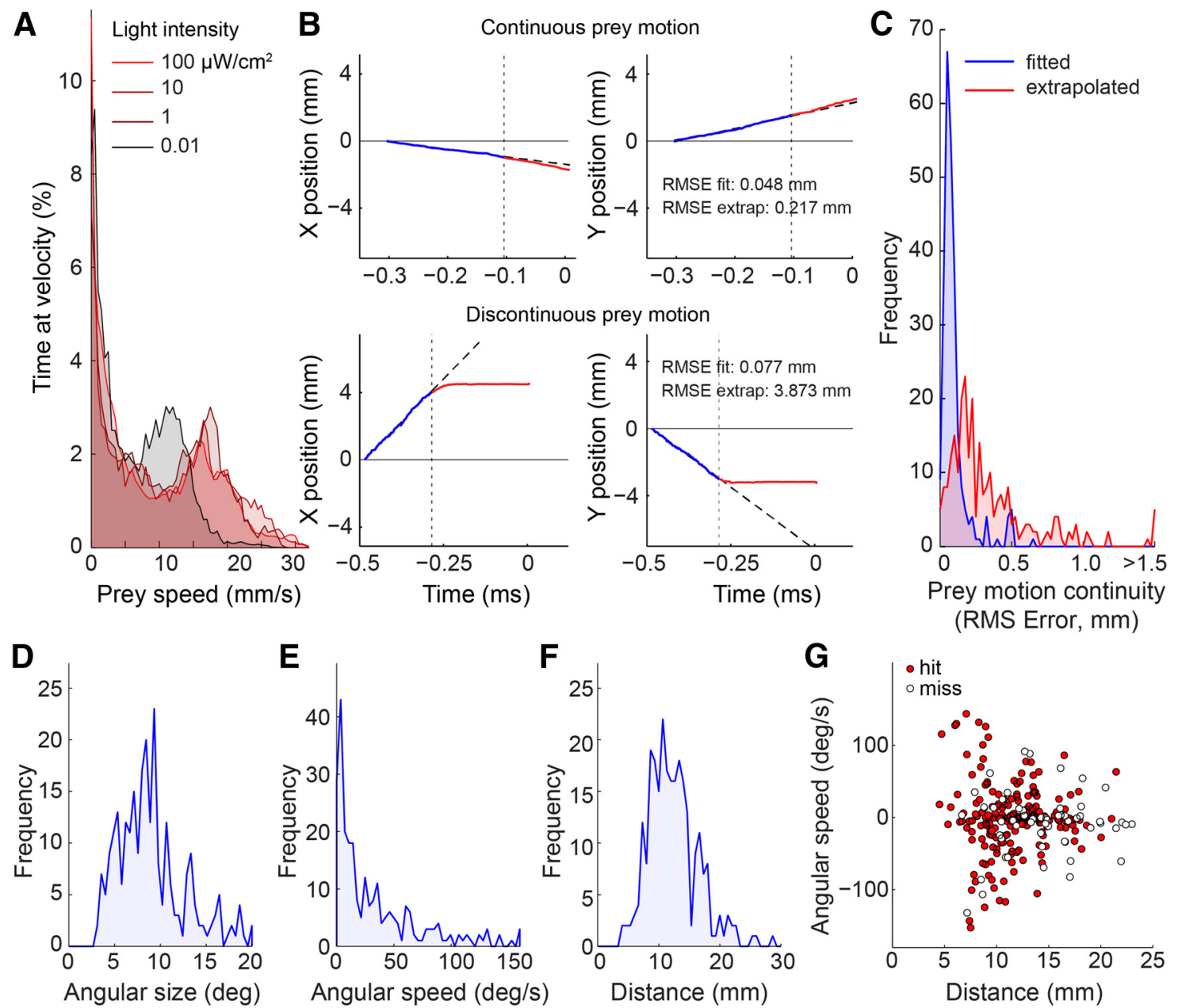

Figure 2. Prey motion trajectories are diverse but often predictable. $A$, Distribution of prey speed at different light levels. At each light level, the distribution was bimodal with a zero and non-zero peak, indicating that flies alternated between stationary and walking periods. $\boldsymbol{B}$, Computation of prey motion continuity for two sample fly trajectories leading up to tongue projection. Prey motion was divided into two time segments relative to capture onset (vertical dotted line): the pre-capture phase ( $200 \mathrm{~ms}$ of motion before capture onset; blue line), and the capture phase (motion during prey capture until the tongue was fully extended; red line). A linear fit (dashed black line) to prey motion in the pre-capture phase was used to predict prey position in the capture phase. Prey motion continuity was defined as the RMS error between the extrapolated fit and the observed prey motion trajectory (red). C, Histogram of motion continuity across all recorded projections. Low RMS error indicates predictable prey motion with little deviation from its pre-capture trajectory. RMS error of the linear fit (blue segment in $\boldsymbol{B}$ ) included for reference. Most trajectories were predictable to $<0.5$ $\mathrm{mm}$ error (a fly is $\sim 3 \mathrm{~mm}$ in length and moves $\sim 3 \mathrm{~mm}$ during a typical capture phase). $\boldsymbol{D}$, Distribution of prey angular size on the salamander retina at the onset of prey capture. $\boldsymbol{E}$, Distribution of prey angular speed at the onset of prey capture. $\boldsymbol{F}$, Distribution of prey distance from the salamander at the onset of prey capture. $\boldsymbol{G}$, Prey distance and angular speed across all projections. Positive and negative values indicate opposite directions of motion. Successful captures (hits, red circles) and failed captures (misses, open circles) are broadly distributed across the parameter space.

projection errors (on average, $7.48^{\circ} \mathrm{SD}$ across all light levels, $0.01-250 \mu \mathrm{W} / \mathrm{cm}^{2}$ ), small systematic dependencies of projection error on either angular speed or size (Leonardo and Meister, 2013) may be obscured in this paradigm.

The range of prey eccentricities (prey angular position with respect to the salamander's visual mid-line) at the time of tongue projection was directly dependent on prey distance. When the prey was near the salamander $(<10 \mathrm{~mm})$, projections were made over a large range of eccentricities, $\sim \pm 20^{\circ}$ (Fig. $3 E$ ). By contrast, when the prey was far from the salamander $(>20 \mathrm{~mm})$, projections were made within a very restricted range of eccentricities, $\pm 5^{\circ}$ of the visual mid-line. Thus, tongue projections are tuned not merely to the distance to the prey but also to its angular offset. Prey eccentricity is not accounted for via a compensatory angle in the tongue projection relative to the head (addressed later in Fig. 4), but instead in the process used to select prey: salamanders did not project to prey with both a large retinal eccentricity and a large distance. Furthermore, when we plotted the average tongue projection error magnitude as a function of prey distance, we found that errors were not constant but varied systematically with distance: more distant prey were hit with lower angular error (Fig. $3 F$ ). At each prey distance, projections were approximately twofold more accurate than strictly required by prey angular size (Fig. $3 F$ ). This apparent safety margin contributes to the high capture success rate at all projection distances. These data show that the salamander targets prey within a specific volume, \pm 3.5 $\mathrm{mm}$ from the mid-line to a depth of $\sim 22 \mathrm{~mm}$. The salamander rarely shot at prey outside this volume. We therefore conclude that the guidance mechanisms for tongue projections are strongly influenced by mechanical constraints of the tongue itself: its maximal length and the angular scaling of the tongue pad with distance.

Next, we asked how accurately tongue projections are controlled in depth: is the tongue always projected to maximal extension, or are projections tailored to prey distance? Tongue extension varied approximately linearly with prey distance up to $20 \mathrm{~mm}$, and saturated $\sim 23.0 \mathrm{~mm}$ (Fig. $3 G, H$ ). At maximal extension, the tongue-tip exceeded the prey by $1.58 \pm 0.15 \mathrm{~mm}$, 

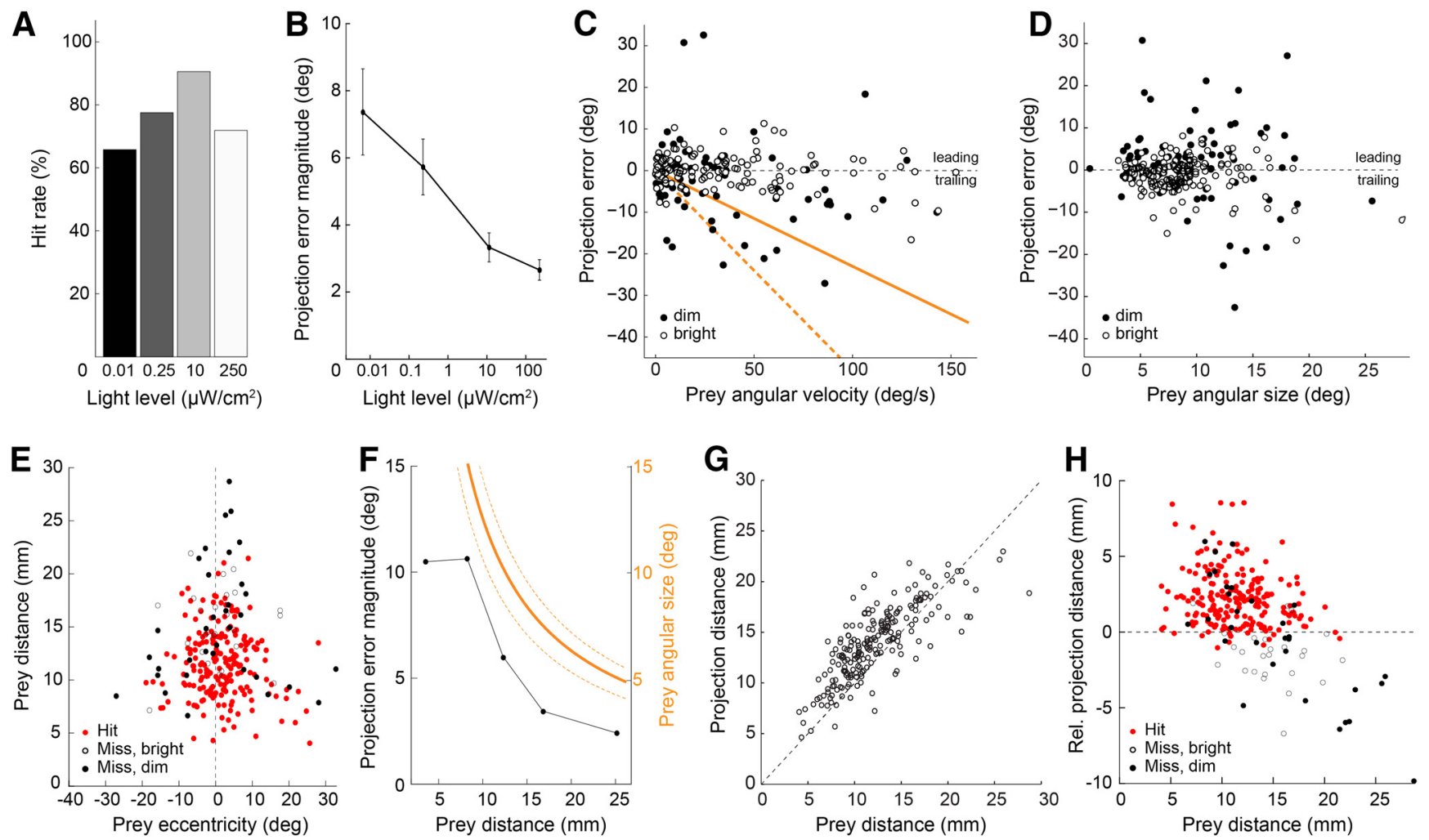

Figure 3. Tongue projection accuracy is robust across a wide range of illumination and prey motion conditions. All panels: $n=268$. A, Prey capture success rate from dim through bright light levels (scotopic through photopic; $4.5 \log _{10}$-unit range). $\boldsymbol{B}$, Magnitude of the angular error across all tongue projections at the light levels in $\boldsymbol{A}$. Data include both hits and misses. $\boldsymbol{C}$, Scatter plot of the projection error as a function of prey angular velocity at the time of projection onset. Orange lines indicate the projection error that would result if the salamander aimed at a delayed prey image with a $230 \mathrm{~ms}$ (solid) and $480 \mathrm{~ms}$ (dashed) visuomotor lag. $\boldsymbol{D}$, Scatter plot of projection error as a function of prey angular size. $\boldsymbol{E}$, Scatter plot of prey distance versus prey eccentricity relative to the salamander's visual mid-line (vertical dashed line) for all recorded projections. Nearby prey evoked tongue projections at eccentricities up to $25^{\circ}$, whereas distant prey evoked projections only when located within a few degrees of the midline. $F$, Comparison of the angular projection error (black) and prey angular size (orange; average size, solid line; maximum and minimum size, orange dashed line) as a function of prey distance. Tongue projection error decreased with increasing prey distance, such that at most distances the angular error was approximately twofold smaller than prey angular size. G, Scatter plot of the tongue projection distance versus prey distance (see Materials and Methods; Fig. 1). $\boldsymbol{H}$, Scatter plot of relative projection distance (tongue tip distance to prey distance). The tongue typically overshot the prey by $\sim 2.5 \mathrm{~mm}$; at the brightest light level, projections increasingly often landed short of the prey (open circles).

which ensured that in most cases the center of the tongue pad contacted the prey. At the light level where the hit rate peaked (10 $\mu \mathrm{W} / \mathrm{cm}^{2}$; Fig. $3 A$ ), projections were extremely accurate in distance and only $3 \%$ of tongue projections fell short of the prey by $>1 \mathrm{~mm}$. At $250 \mu \mathrm{W} / \mathrm{cm}^{2}$, however, this fraction rose to $20 \%$ (Fig. $3 H)$. Thus, an increased rate of distance errors, where the tongue projection fell short of the prey, reduced capture success in bright light (Fig. 3A) despite an increase in angular accuracy (Fig. 3B). Why bright light caused these short projections is not clear. In contrast, reduced capture success in dim light reflected increased error in both distance and azimuthal angle (Fig. $3 B, H$ ).

In sum, salamander tongue projections were highly accurate over a wide range of prey distances, speeds, and light levels. The angular velocity data in particular further the notion that the underlying guidance of the tongue is based on a prediction of future prey location (Fig. 3C).

\section{Ballistic head turns increase the prey capture-associated sensorimotor delay}

If the salamander uses a predictive strategy to aim the tongue, then a crucial parameter is the time interval over which that prediction is made. This time interval should be determined primarily by the sensorimotor delay in the salamander's nervous system. The response latency of amphibian retinal ganglion cells to small targets is well known and ranges from $\sim 0.1 \mathrm{~s}$ in mid photopic (Leonardo and Meister, 2013) to $\sim 1 \mathrm{~s}$ in scotopic light levels (Aho et al., 1993). What is less well established are the motor delays when pursuing freely moving prey, specifically those originating from rotation of the head and the tongue projection itself.

The salamander tended to project at prey as they crossed the visual mid-line (Fig. 3E). However, it rarely left the timing of this crossing to the prey. Instead, rapid head turns that centered the prey with respect to the visual mid-line were a key component of the prey capture process. We distinguished two types of head turn: orienting and projection head turns, defined by the absence or presence, respectively, of an immediately following tongue projection ( $<15 \mathrm{~ms}$ delay; see Materials and Methods). Orienting head turns (typically one or two per capture trial) occurred when the prey entered a region $\pm 50^{\circ}$ from the visual mid-line (Fig. $4 A$ ). These head rotations brought the prey image toward the center of the salamander's visual field, but not necessarily in precise alignment with the mid-line $\left( \pm 18.1^{\circ}\right.$ of mid-line after rotation). This contrasts with projection head turns, which occurred only when the prey was already within $\pm 30^{\circ}$ of the visual mid-linethe region of visual space prominently covered by the binocular field of the two eyes (Fig. 4A, inset) (Roth, 1987). At the completion of a projection head turn, prey were centered on the visual mid-line more precisely compared with orienting head turns (Fig. $4 A, \pm 11.8^{\circ}$ of mid-line). More than $70 \%$ of tongue projections followed a detectable head turn (see Materials and Meth- 
A

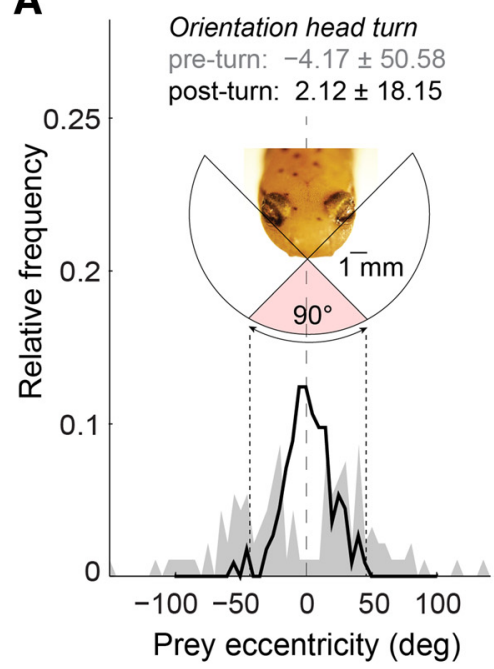

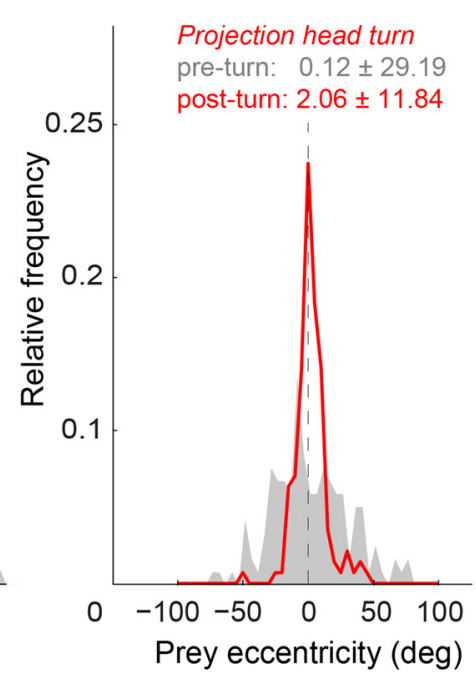
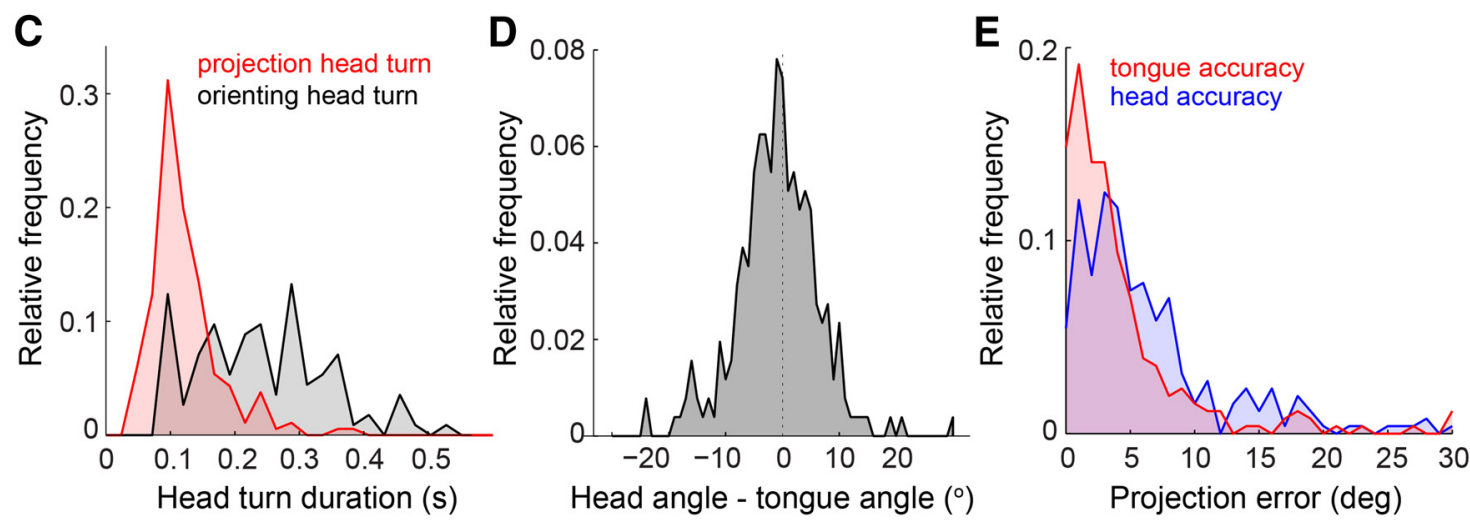

Figure 4. Most tongue projections are preceded by a fast and accurate, fixed-duration head turn. Head turns were defined as head rotations exceeding 5 degrees in amplitude and $10^{\circ} / \mathrm{s}$ in angular velocity. All panels: $n=268$. A, Left, Orienting head turns (head turns not followed by a tongue projection) brought the prey image from the periphery of the salamanders field of view into the binocular zone. Right, Projection head turns (head turns immediately followed by a tongue projection; see Materials and Methods) began with the prey within the binocular field of view and ended with the prey tightly centered around the visual mid-line. $\boldsymbol{B}$, Angular velocity of all recorded orienting and projection head turns. Arrow indicates one $x$-shifted data point with head turn amplitude of $124^{\circ}$. C, Distribution of orienting and projection head turn durations. Projection head turns were less variable and had a significantly shorter duration compared with orienting head turns (123 \pm $4.0 \mathrm{~ms}$ vs $264 \pm 16 \mathrm{~ms}$, mean \pm SEM, $n=186, n=113$; unpaired $t$ test, $p<0.0001$ ). D, Distribution of the angle between the head direction and tongue direction relative to the visual mid-line (see Materials and Methods). The tongue was projected along the visual midline on average, but deviations up to $\pm 5^{\circ}$ were common. $E$, Distribution of tongue projection error and head orientation error, relative to prey position.

ods), indicating that the projection head turn is an integral part of the tongue projection behavior.

Projection and orienting head turns differed not only in their angular precision, but also in their speed. Projection head turns were rapid, with angular velocities up to $500^{\circ} / \mathrm{s}$ (average $150 \pm$ $8 \%$ SEM; $n=186$ ), whereas orienting head turns were relatively slow with an average angular velocity of $87 \pm 4.6^{\circ} / \mathrm{s}$ SEM $(n=$ 113; Fig. $4 B$ ). Projection head turns were also faster and less variable in duration than orienting head turns $(p<0.0001, t$ test; mean duration projection turn $123 \pm 4.0$ ms SEM, mean duration orienting turn $264 \pm 16 \mathrm{~ms}$ SEM; Fig. $4 C$ ). The strong correlation between amplitude and speed of the projection head turn (slower speeds for smaller turns, faster speeds for larger turns), and its invariance across light levels (data not shown) caused the duration of each head turn to be $\sim 120 \mathrm{~ms}$, regardless of prey eccentricity. The conserved head turn duration resembles what is seen during human saccades (Zangemeister et al., 1981), suggesting that they, too, are ballistic movements.

While some salamander species can launch the tongue at a considerable angle relative to the head (Roth, 1976), three-lined salamanders showed only modest capability in this respect. On average, the mean angle between the direction of the tongue and the direction of the head was $-0.96^{\circ}$ (demonstrating absence of a significant bias to left or right of the midline) with an SD of 7.19 , which represents the extent to which the tongue was angled relative to head orientation (Fig. 4D). Nevertheless, the angular error between the tongue direction and the prey was significantly lower than the angular error between the head direction and the prey $\left(4.30 \pm 6.14^{\circ}\right.$ vs $6.29 \pm 5.87^{\circ} ; p=0.0002, n=256$; Fig. $\left.4 E\right)$, suggesting that the salamander's control of the tongue-angle relative to the head reduces the projection error that would result from that set by the head-angle alone.

Tongue projection kinematics have been studied for projections to dropped prey (Deban et al., 2007); here, we examined them to freely moving prey. The average peak velocity of the tongue across all projections and all animals was $2.3 \pm 0.6 \mathrm{~m} / \mathrm{s}$ $\operatorname{SEM}(n=268$; Fig. $5 A, B)$. Comparison of the tongue movement across all projections, grouped into short-, medium-, and longrange projections (Fig. 5A), showed that the kinematics of the tongue were independent of prey distance. Trial-to-trial projection distance thus appeared to be set by the timing of a motor command to stop the motion of the tongue and achieve the de- 
A

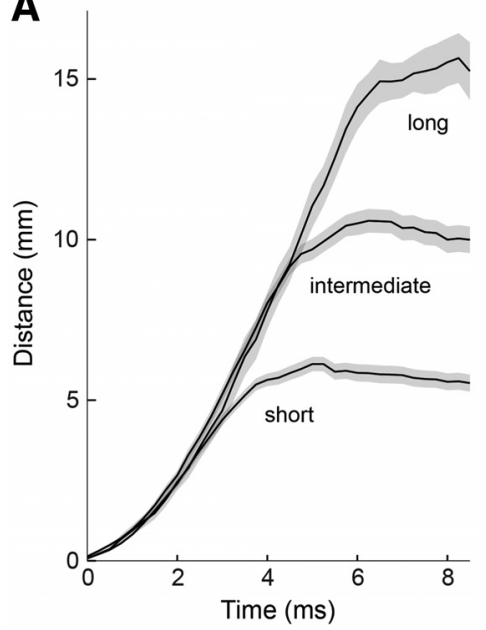

B

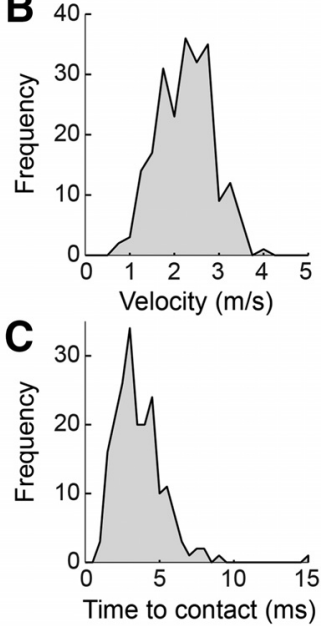

Figure 5. Tongue projections are rapid and ballistic. All panels: $n=268$. A, Movement of the tongue tip during all recorded projections. Projections were grouped into three projection distance categories (short, middle, long) by dividing the range of projection distances into three equal-sized bins. Tongue motion was largely independent of prey distance until deceleration just before prey contact. $\boldsymbol{B}$, Histogram of peak tongue velocity for all recorded projections, from 6 different salamanders. C, Time to contact between tongue and prey, for all projections.

sired strike distance, rather than one to expel the tongue with more or less force (Deban and Dicke, 1999). Prey at a range of 5 $\mathrm{mm}$ were struck in $\sim 4 \mathrm{~ms}$, with an additional millisecond for each additional $5 \mathrm{~mm}$ of distance. Thus, the typical time to contact was $\sim 5 \mathrm{~ms}$ (Fig. $5 \mathrm{C}$ ). These data demonstrate that tongue projections were ballistic and launched with stereotyped kinematics, while projection distance was controlled for each prey.

Although executed rapidly and ballistically, the projection head turn and tongue extension added $\sim 130 \mathrm{~ms}$ to the total sensorimotor delay ( $123 \mathrm{~ms}$ for the head turn and $5 \mathrm{~ms}$ for the tongue projection; Figs. $4 C, 5 C$ ). Combined with a retinal response latency of at least $100 \mathrm{~ms}$ (Leonardo and Meister, 2013), the cumulative visuomotor latency from prey detection to prey capture is at least $\sim 230 \mathrm{~ms}$. During this time, the prey used here (Drosophila melanogaster), at an average angular size of $9^{\circ}$ and an angular speed of $34^{\circ} / \mathrm{s}$, would move as much as one body length (Fig. 2D,E). Given these motion statistics, if the visuomotor delays were uncompensated they would yield an average projection error of $8^{\circ}$, more than twofold larger than observed $\left(\sim 3^{\circ}\right.$, Fig. $3 B$ ). We conclude that prey motion statistics (Fig. 2), salamander sensorimotor delays (for retina latency, see Figs. 4, 5; Leonardo and Meister, 2013), and tongue projection accuracy as a function of prey speed (Fig. 3C) and luminance (Fig. 3B), are consistent with predictive guidance of the tongue and constrain its underlying mechanisms.

\section{Guidance law underlying tongue projections}

While a variety of predictive models might be used to estimate future prey position, the salamander must act with both speed and precision. Because most prey trajectories are continuous and approximately linear, linear extrapolation is one of the simplest and fastest strategies that could accurately predict future prey position (Fig. 2B,C). In this section, we test the sufficiency of linear extrapolation to explain the predictive guidance used by the salamander to aim its tongue (Fig. 1D). For successful projections, this will match the prey's position; for unsuccessful projections, it will give an error relative to the prey's position but still match the location of the tongue. We assessed the quality of the fit
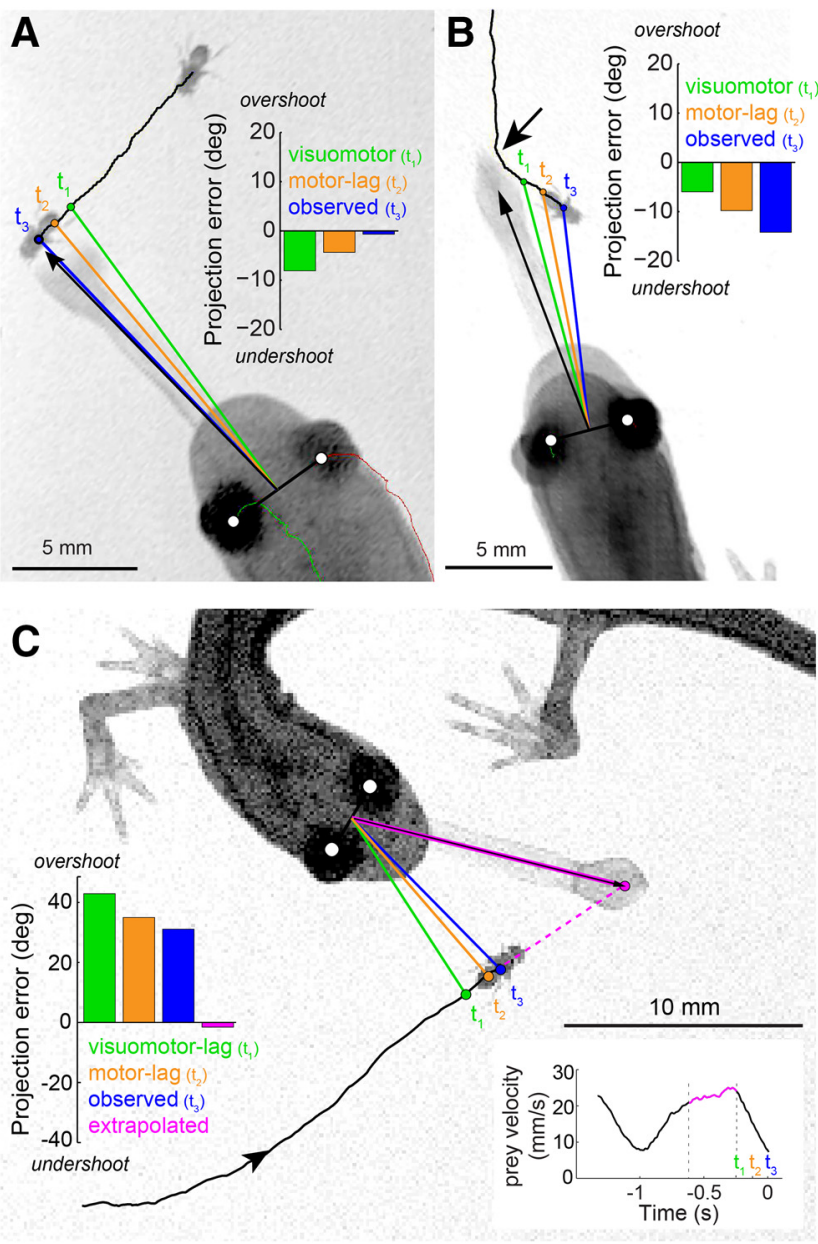

Figure 6. Salamander tongue projections are aimed at a predicted prey location. $A$, Example of a projection aimed at a prey that moved with near-constant velocity on a continuous trajectory. The visuomotor-lag model would target the prey at position $\mathrm{t}_{1}$ (i.e., one sensorimotor latency behind its final position). The motor-lag model would target prey at position $\mathrm{t}_{2}$, one motor delay behind its final position at the onset of the head turn. $t_{3}$ marks the measured final prey position at the time of tongue projection. Evaluation of the relative angle between tongue and prey at $t_{1}, t_{2}$, and $t_{3}$ (bar plot, inset) shows that the tongue lands nearest to the prey at $t_{3} . \boldsymbol{B}$, Example of a projection aimed at a prey that moved with near-constant velocity on a discontinuous trajectory. Visuomotor-lag, motor-lag, and final prey position marked as in $\boldsymbol{A}$. The tongue lands at a location that is distant from the final prey position at $t_{3}$, but where the prey would have been had its trajectory remained continuous (dim light conditions; arrow indicates turn $\sim 300 \mathrm{~ms}$ before $\mathrm{t}_{3}$ ). C, Example of a projection aimed at a prey that stopped moving at the onset of the projection head turn $\left(\mathrm{t}_{2}\right)$. The tongue lands well ahead of the prey. Extrapolation of the prey's motion before the head turn (bottom right inset; magenta) predicts the prey position after the head turn. This extrapolated position is consistent with where the tongue was projected and considerably different from both the observed final prey position $\left(\mathrm{t}_{3}\right)$ and the visuomotor-lag $\left(\mathrm{t}_{1}\right)$ and motor-lag $\left(\mathrm{t}_{2}\right)$ models.

by comparing the observed tongue position with that expected from an extrapolation model, compensating for both visual and motor delays, as well as to what would be attained if the salamander used a motor-lag model and compensated for the visual delay but not the motor one, or if the salamander used the visuomotorlag model and compensated for neither delay (Fig. 1D).

We begin by developing the extrapolation model over three specific examples and then expand the analysis to test whether this strategy generalizes across all recorded projections. In the first example (Fig. $6 A$ ), the salamander projects at a prey moving at nearly constant speed and fixed bearing. If the salamander aimed at the prey position $100 \mathrm{~ms}$ (one visual latency) before the onset of the projection head turn or at the onset of the head turn, 
projection errors would have been large, lagging the prey by $8.1^{\circ}$ and $4.4^{\circ}$, respectively. The observed projection error to the final prey position, however, was much smaller; just $0.7^{\circ}$. In the second example $\left(0.003 \mathrm{~mW} / \mathrm{cm}^{2}\right.$ ambient illumination; Fig. $\left.6 B\right)$, the prey turned $\sim 300 \mathrm{~ms}$ before the onset of the projection head turn. Here, the tongue was projected to a location that was far from the final prey position, but close to a portion of its trajectory before the turn (Fig. $6 \mathrm{~B} ; 14^{\circ}$ error to final prey position). This second example is consistent with extrapolation, but also with a lagged guidance model that aims at a delay imaged of the prey (Fig. 1D). However, the third example is definitive. In Figure 6C, the prey stopped walking at the onset of the head turn. In this case, the tongue landed well ahead of the fly, at a phantom location that the prey never visited, nor was even close to. However, the final position of the tongue is consistent with where the prey would have gone, had it not stopped. Indeed, fitting a simple linear extrapolation model to this event, based on the prey's velocity immediately before the head turn, accurately predicts where the tongue was projected (Fig. 6C).

The last two examples provide explicit support for linear extrapolation (Fig. $6 B, C$ ). Because the tongue is projected to a physical location never occupied by the fly, these examples argue against a first-order "bug detector"-type model where visual stimulation of a specific photoreceptor location on the retina triggers a projection to the corresponding location in visual space (Lettvin et al., 1959). These data instead suggest that the salamander aims the tongue not at the target as conveyed by its photoreceptor image but at a predicted future position, computed from the movement history of its image on the retina.

If predicted prey position underlies tongue guidance, then it should account for tongue position across all projections better than nonpredictive models. To test this, we compared the tongue position estimate from the extrapolation model to the two nonpredictive models in which the salamander aimed at the lagged visual images of the prey (Fig. 6). The visuomotor-lag model assumed that the salamander aimed at the prey position one visual latency before the projection head turn (100 ms for bright light; $350 \mathrm{~ms}$ in dim light) (Aho et al., 1993), and thus compensated for neither the visual nor the motor delays. The motor-lag model assumed the salamander aimed at the prey at the start of the head turn, and compensated for the visual but not the motor delay. The extrapolation model estimated future prey position via linear extrapolation and required two parameters, an integration time and a delay, which we obtained from the behavior data. The integration time $(\tau)$ defined the duration over which prey velocity was assessed; the delay $(\Delta t)$ defined how far back in time this integration window ended relative to the onset of prey capture (Fig. 7A). With these parameters, the predicted future prey position was determined by fitting a line to the prey position during the integration time window and extrapolating this line forward in time up to the time of maximal tongue extension (Fig. $7 A$, schematic).

Best estimates for $\tau$ and $\Delta t$ were obtained through an exhaustive search of the parameter space across all recorded projections ( $\tau=10-750 \mathrm{~ms} ; \Delta t=0-1000 \mathrm{~ms}$ ). For each combination of $\tau$ and $\Delta t$, we calculated the tongue model error by comparing the extrapolated prey location (where the model would direct the tongue) to the observed tongue location (where the salamander projected the tongue). Some projections constrained parameter values more than others. For prey moving with near-constant velocity along a straight trajectory, nearly all combinations of $\tau$ and $\Delta t$ yield the same extrapolated prey location and the expected error across the parameter space is approximately constant (e.g.,
Fig. 7A). In contrast, for curved trajectories, only a small number of $(\tau, \Delta t)$ combinations will result in a projection consistent with the observed tongue angle, producing a concave error surface with a distinct minimum (e.g., Fig. 7B). By averaging the error surfaces across all projections and locating the global minimum of this averaged error surface, we obtained estimates for integration time and delay that maximized performance of the extrapolation model. Because light adaptation changes the temporal kinetics of photoreceptors and postsynaptic visual responses, we should expect $\tau$ and $\Delta t$ to change with ambient light level. Therefore, to obtain parameter fits to the behavior data, projections were divided into bright and dim light-level brackets (for details, see Materials and Methods), and their average error surfaces were computed separately.

The integration time that minimized the predicted tongue error was $\sim 175 \mathrm{~ms}$ across both light levels, whereas the delay decreased from $250 \mathrm{~ms}$ in dim light to $100 \mathrm{~ms}$ in bright light (Fig. $7 C, D$; see Materials and Methods). Using these values, we assessed how well the extrapolation model predicted tongue location across all projections, and compared its efficacy against the visuomotor-lag and motor-lag models. The final position of the prey is not used in this analysis; instead, we asked how well prior positions of the prey can account for the final position of the tongue. We found that the extrapolation model predicted the observed tongue location better than both the visuomotor-lag and the motor-lag model (Fig. $7 C, D$ ). In bright light, the average prediction error for tongue location from the extrapolation model was $2.8 \pm 2.8^{\circ}$, versus $7.9 \pm 8.0^{\circ}$ and $5.0 \pm 5.1^{\circ}$ for the visuomotor-lag and motor-lag models, respectively. Similar results were obtained in dim light (visuomotor-lag model, $9.7 \pm$ 11.7; motor-lag model, $7.5 \pm 9.8$; extrapolation model, $6.2 \pm$ $\left.7.7^{\circ}\right)$. Errors in dim light were higher than those in bright light for all models, which would be expected because increased visual latency gave increased opportunity for unexpected prey motion during the longer time interval before tongue projection.

Overall, the extrapolation model predicted tongue position as accurately as the final position of the prey (Fig. $7 C, D$ ). This is consistent with Figure $2 C$, as most prey trajectories were continuous and predictable on a short time scale by linear extrapolation. However, a small subset of prey trajectories was highly discontinuous (e.g., Fig. $6 \mathrm{~B}, \mathrm{C}$ ); and in several of these cases, we found that the tongue position was predicted better by the extrapolation model than by the actual prey position at the time of maximal tongue extension (Fig. 7E). In other words, when the tongue was aimed badly, its error was often still consistent with the extrapolation model. Thus, we conclude that linear extrapolation over a biologically plausible integration time window can account for salamander tongue projection accuracy at the level of the accuracy of the behavior.

\section{Discussion}

For behavior to be accurate in the face of sensorimotor delays, prediction is necessary. We investigated how such predictive computation is used by the salamander during tongue projections to moving prey. There are four major results to our study. First, salamander tongue projections are highly accurate and robust to large variations in light level, prey angular size, and prey angular speed (Fig. 2). Second, there are significant sensorimotor delays underlying this accuracy: $\sim 100 \mathrm{~ms}$ from phototransduction through retinal ganglion cell response latency, which increases substantially with decreasing light level (Aho et al., 1993; Leonardo and Meister, 2013), $123 \mathrm{~ms}$ from the projection head turn (Fig. 4), and $5 \mathrm{~ms}$ from the tongue projection (Fig. 5). While 

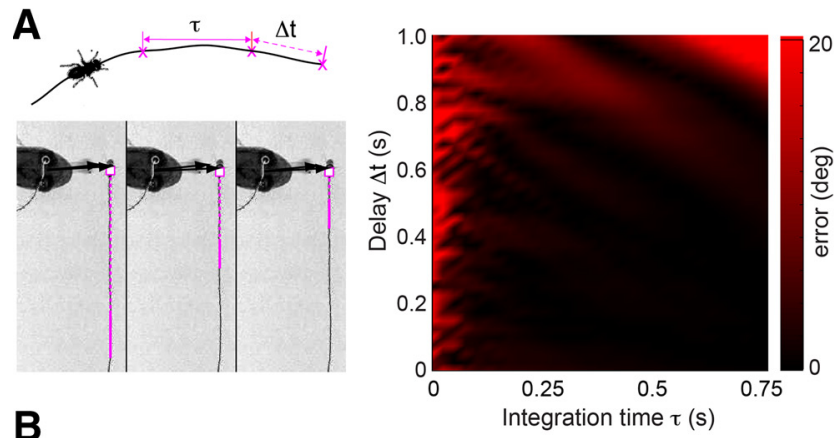

B
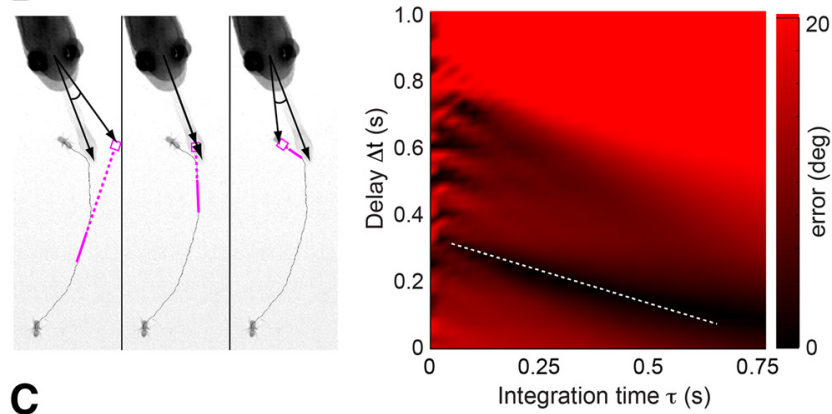

C
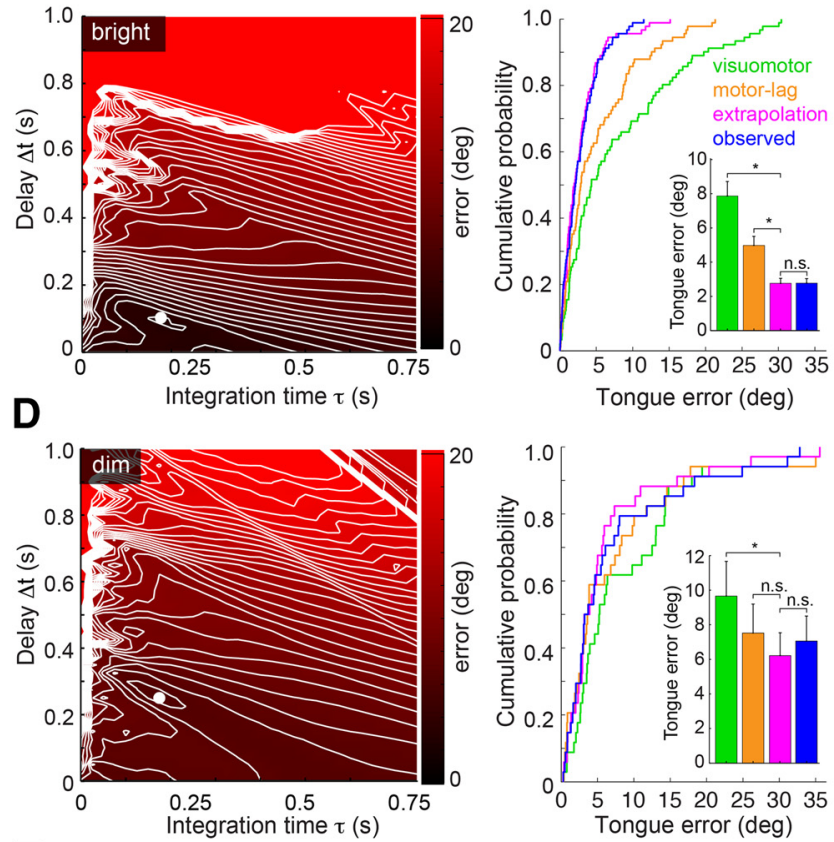

$\mathbf{E}$
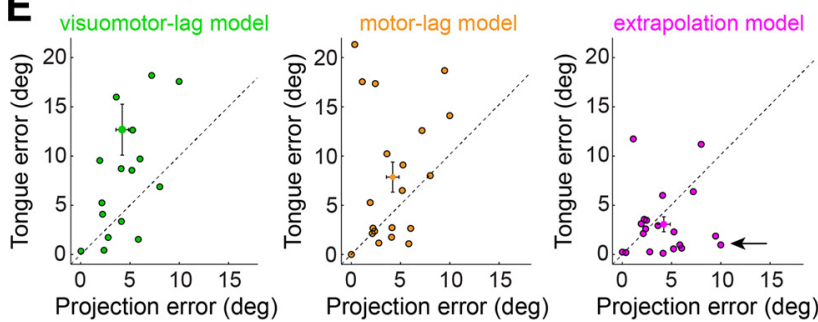

Figure 7. Linear extrapolation of prey trajectory predicts tongue position to within behavioral variability. $\boldsymbol{A}$, Left, Top, Schematic representation of extrapolation model, showing the prey movement, integration window $(\tau)$, and visual latency $(\Delta t)$ used to compute the predicted prey location. Left, Bottom, Example of a tongue projection to a prey with near-constant velocity on a continuous trajectory. Panels represent the extrapolated prey trajectory for three different integration time $(\tau)$ and delay $(\Delta t)$ combinations (magenta line indicates integration time window). For each combination, this continuous prey trajectory results in the same predicted future prey location (magenta circle). Right, Surface plot of tongue position error (angular difference between the tongue and extrapolated prey location) demonstrating that most the tongue projection time is negligible relative to the escape time of a fly ( $100 \mathrm{~ms}$ ) (Card and Dickinson, 2008a), the visual latency and head turn are not. Third, the aggregate visuomotor delay ( $\sim 230 \mathrm{~ms}$ in bright light, larger in dim light; Figs. 4, 5) and fly walking speed $(34 \%$ s, Fig. 2$)$ together imply that a predictive mechanism must guide tongue projections or the average tongue projection error $\left(\sim 3^{\circ}\right.$; Fig. $\left.3 B\right)$ would be considerably larger. Fourth, the salamander's tongue projections are consistent with a simple predictive model in which linear extrapolation is used to estimate the prey's expected location at the moment of maximal tongue extension (Figs. 6, 7). In implementing this model, the salamander must have an internal representation of the prey's velocity, and targeting must account for the size and projection length of the tongue (Fig. 3C,E). Linear extrapolation of the prey position significantly expands the range of prey motions and environmental conditions under which the salamander can hunt effectively.

Our results allow a compact description of the algorithmic steps underlying amphibian prey capture that highlights the precise computational problems that must be solved within the neural circuitry. Amphibian prey capture comprises two distinct phases: a sensory phase and a motor phase. During the sensory phase, the salamander generally remains motionless until it detects prey and computes the relevant targeting parameters to be used in the pursuit phase. When the prey leaves the $\sim 90^{\circ}$-wide region of binocular visual overlap, a relatively slow orienting head turn is made to bring the prey back toward the visual midline (Fig. 4). Unlike the retinas of birds and most higher mammals, the salamander retina is isotropic, lacking an anatomically specialized region for increased spatial resolution (Linke et al., 1986). Isotropy obviates the need for saccades and pursuit eye movements (Martinez-Conde and Macknik, 2008): prey can be tracked with constant spatial acuity across the retina. A first advantage is that the salamander can remain motionless longer, reducing the risk of breaking camouflage. A second advantage is

parameter combinations for continuous prey trajectories give a similar error. $\boldsymbol{B}$, Left, Discontinuous prey trajectories yield diverse extrapolated prey positions as a function of $\tau$ and $\Delta t$. Right, Surface plot of tongue position error demonstrating that the discontinuous trajectory generates minima at a limited set of $\tau$ and $\Delta t$ combinations (dashed white line). C, Tongue position error surface and extrapolation model performance for bright light projections $\left(2-250 \mu \mathrm{W} / \mathrm{cm}^{2}\right.$; see Materials and Methods). Left, Error surface showing a strong minimum in extrapolation model error ( $\tau=175 \mathrm{~ms}, \Delta t=100 \mathrm{~ms}$ ). Right, Cumulative probability distribution function of errors for the visuomotor-lag, motor-lag, extrapolation model, and observed projection errors in predicting tongue position. The visuomotor-lag model aimed at the prey $100 \mathrm{~ms}$ before the head turn. The motor-lag model aimed at prey at the moment of the head turn. The extrapolation model has considerably lower error than either nonpredictive model, and estimated the tongue location approximately equally well as the observed prey position (inset, bar plot). Significance for paired $t$ tests: visuomotor-lag versus extrapolation, $p<0.0001$; motor-lag versus extrapolation, $p<0.0001$; extrapolation versus observed, $p=0.96$. $\boldsymbol{D}$, Tongue position error surface and performance for dim light projections $\left(0.01-0.2 \mu \mathrm{W} / \mathrm{cm}^{2}\right.$; see Materials and Methods). Left, Error surface showing a strong minimum in extrapolation model error at ( $\tau=250 \mathrm{ms,}$ $\Delta t=100 \mathrm{~ms}$ ). Right, Cumulative probability functions as in C. The visuomotor-lag mode aimed the prey $350 \mathrm{~ms}$ before the head turn. The motor-lag model aimed at prey at the moment of the head turn. The extrapolation model has considerably lower error than either nonpredictive model and often estimates the tongue position better than the observed final prey position (inset, bar plot). Significance for paired $t$ tests: visuomotor-lag versus extrapolation, $p=0.046$; motor-lag versus extrapolation, $p=0.27$; extrapolation versus observed, $p=0.32$. ${ }^{*}$ indicates significant differences; n.s., not significant. $\boldsymbol{E}$, Scatter plots of observed projection error (angle between the measured final positions of tongue and prey) versus the tongue model error (angle between measured tongue position and tongue position computed from each model). Errors are plotted in absolute value, using data from the 20 most discontinuous prey trajectories. Arrow indicates tongue positions that are explained better by extrapolated prey location than actual prey location. 
that during the sensory phase the eyes, head, and tongue all share the same coordinate system. This vastly simplifies the prey capture problem compared with other species in which head and/or eyes move relative to the body (Mischiati et al., 2015) and require continuous dynamic mapping of multiple coordinate systems.

Once initiated, the motor phase of the capture behavior is rapid and inexorable. When the prey is within the binocular zone, the salamander makes a ballistic head turn that brings the prey precisely to the mid-line (Fig. $4 A, C$ ) and culminates in a launch of the tongue and capture of the prey. The tongue projection is made with maximum acceleration and velocity over a brief period of time. Projections are ballistic, with a distinct absence of feedback control while the motion is executed (Fig. 3). There is little azimuthal control over the angle at which the tongue leaves the mouth (Fig. 4); consequently, the salamander controls the azimuthal angle of the projection primarily by setting the headbody angle. The combined motion of the head and tongue allow the salamander to target prey in a small volume $\sim 2-4 \mathrm{~mm}$ wide and $20 \mathrm{~mm}$ long, centered on the visual mid-line of the head (Fig. 3E).

Our data show that azimuthal control of the tongue is fundamentally based on motion extrapolation of the prey's trajectory, which begins shortly before the head turn onset. In our working model (Fig. 7), at bright light levels, $\sim 100 \mathrm{~ms}$ before the head turn, the salamander assesses the prey's bearing over a $175 \mathrm{~ms}$ window and then uses these data to extrapolate the prey's position forward linearly, over both the visual and motor latencies $(\sim 230 \mathrm{~ms})$, to predict the prey's location at the time of maximal tongue extension. Whether this extrapolation accounts for prey acceleration is not known. Tongue projection errors were largely independent of prey angular speed, ruling out that the projection merely leads the prey by a fixed "average" angle. Instead, each projection is adjusted to the angular speed and range of the individual prey (Fig. 3 C). This implies that the salamander computes both of these parameters. Whether depth control relies on extrapolation remains unclear. Because prey position does not vary strongly in depth within the extrapolation time window, its impact would be subtle.

The effect of extrapolation on capture success is considerable. In bright light, if the salamander were to shoot nonpredictively at the delayed prey image location on the retina, it would have an average error of $\sim 8^{\circ}$ in bright light (and likely miss) versus the $3^{\circ}$ that it attains with extrapolation (Fig. $7 C, D$, right panel). With decreasing light level, the parameters of the extrapolation model shift to larger values and projection accuracy deteriorates (Figs. $3 B, 7)$, consistent with known mechanisms intrinsic to the amphibian retina, in particular slower responses in dim light (Aho et al., 1993). As the salamander averages the fly's bearing over longer and more delayed time intervals, it must predict further forward in time and becomes increasingly prone to projection errors induced by unexpected fly turns.

Each step in the prey capture algorithm must be implemented by the nervous system. Here, we briefly consider constraints on the neural mechanisms underlying the control of tongue projection depth and azimuthal angle. We know that the salamander uses distance information to guide the tongue projection, as tongue projection distance depends on prey distance (Fig. 3G, $H$ ). Distance information might be obtained from the disparity between the images from the two eyes (Collett, 1977). If such binocular visual input is essential, then our evidence that the head turn is ballistic implies that the salamander cannot make accurate projections to prey located outside the binocular visual field. Consistent with this, the salamander rarely attempts projection to targets of $>50^{\circ}$ eccentricity (Fig. $4 B$ ), the approximate boundary of the binocular field as suggested by anatomical studies (Roth, 1987). Moreover, when such eccentric projections are attempted, most result in misses ( 3 of 4 in our dataset). Thus, binocular information appears necessary for accurate projections. However, the increased rate of depth errors in bright light suggests the control of depth relies on more than disparity alone. Increased depth of field from pupillary constriction, or other forms of light adaptation, may confound mechanisms of depth detection.

In contrast with depth control, azimuthal control can be achieved by extrapolation based on information from a single eye and may occur within the retina itself. The most obvious candidate for the neural basis of the salamander's azimuthal motion extrapolation is the network of fast-OFF retinal ganglion cells known to exhibit motion anticipation (Berry et al., 1999). Through the nonlinear interaction of excitatory and inhibitory synapses (Johnston and Lagnado, 2015), these cells act as a population to shift the neural image of a moving target forward along its trajectory in a velocity-dependent manner. The range of target angular sizes and speeds over which this neural extrapolation works (Leonardo and Meister, 2013) is well matched to the behavioral range of the salamander shown here (Fig. 3). However, it is unknown how this fast-OFF cell population performs in dim light (e.g., Figs. $2 A, 6 C$ ), and extrapolation circuitry may be located after the retina, for example in the optic tectum. To definitively link extrapolation in the salamander's behavior to the fast-OFF cell circuitry requires demonstrating that the dynamics of the neural model (Leonardo and Meister, 2013), including its subtle biases for target size, speed, and acceleration, contribute to the behavior. This is difficult to assess in the present dataset, with its complex naturalistic trajectories, and is better studied under conditions of precisely controlled prey motion.

Our analysis of salamander prey capture behavior under naturalistic conditions demonstrates unequivocally that prediction underlies guidance of the tongue. This prediction must be based on a model of how prey move, and the projection volume accessible by the tongue. Although we cannot rule out more sophisticated mechanisms, linear extrapolation provides a parsimonious description of the behavior with a level of accuracy equal to the observed projection accuracy, with residual variability likely due to inherent biological noise in the nervous and motor systems. These results open the door to further scrutiny of the neural substrate of prediction at the level of the experimentally accessible retina and optic tectum.

\section{References}

Aho AC, Donner K, Helenius S, Larsen LO, Reuter T (1993) Visual performance of the toad (Bufo bufo) at low light levels: retinal ganglion cell responses and prey-catching accuracy. J Comp Physiol 172:671-682. CrossRef Medline

Berry MJ 2nd, Brivanlou IH, Jordan TA, Meister M (1999) Anticipation of moving stimuli by the retina. Nature 398:334-338. CrossRef Medline

Boeddeker N, Egelhaaf M (2003) Steering a virtual blowfly: simulation of visual pursuit. Proc Biol Sci 270:1971-1978. CrossRef Medline

Boeddeker N, Kern R, Egelhaaf M (2003) Chasing a dummy target: smooth pursuit and velocity control in male blowflies. Proc Biol Sci 270:393-399. CrossRef Medline

Brenner E, Smeets JB (2000) Motion extrapolation is not responsible for the flash-lag effect. Vision Res 40:1645-1648. CrossRef Medline

Card G, Dickinson M (2008a) Performance trade-offs in the flight initiation of Drosophila. J Exp Biol 211:341-353. CrossRef Medline

Card G, Dickinson MH (2008b) Visually mediated motor planning in the escape response of Drosophila. Curr Biol 18:1300-1307. CrossRef Medline 
Collett T (1977) Stereopsis in toads. Nature 267:349-351. CrossRef Medline

Deban SM, Dicke U (1999) Motor control of tongue movement during prey capture in plethodontid salamanders. J Exp Biol 202:3699-3714. Medline

Deban SM, O’Reilly JC, Dicke U, van Leeuwen JL (2007) Extremely highpower tongue projection in plethodontid salamanders. J Exp Biol 210: 655-667. CrossRef Medline

de Rugy A, Marinovic W, Wallis G (2012) Neural prediction of complex accelerations for object interception. J Neurophysiol 107:766-771. CrossRef Medline

Dicke U, Roth G (1994) Tectal activation of premotor and motor networks during feeding in salamanders. Eur J Morphol 32:106-116. Medline

Etchells PJ, Benton CP, Ludwig CJ, Gilchrist ID (2010) The target velocity integration function for saccades. J Vis 10:7. CrossRef Medline

Fisk JD, Goodale MA (1988) The effects of unilateral brain damage on visually guided reaching: hemispheric differences in the nature of the deficit. Exp Brain Res 72:425-435. Medline

Franklin DW, Wolpert DM (2011) Computational mechanisms of sensorimotor control. Neuron 72:425-442. CrossRef Medline

Hartline DK, Colman DR (2007) Rapid conduction and the evolution of giant axons and myelinated fibers. Curr Biol 17:R29-R35. CrossRef Medline

Haselsteiner AF, Gilbert C, Wang ZJ (2014) Tiger beetles pursue prey using a proportional control law with a delay of one half-stride. J R Soc Interface 11:20140216. CrossRef Medline

Johnston J, Lagnado L (2015) General features of the retinal connectome determine the computation of motion anticipation. eLife 4:e06250. CrossRef Medline

Kawato M (1999) Internal models for motor control and trajectory planning. Curr Opin Neurobiol 9:718-727. CrossRef Medline

Land MF, McLeod P (2000) From eye movements to actions: how batsmen hit the ball. Nat Neurosci 3:1340-1345. CrossRef Medline

Leonardo A, Meister M (2013) Nonlinear dynamics support a linear population code in a retinal target-tracking circuit. J Neurosci 33:16971-16982. CrossRef Medline

Lettvin JY, Maturana HR, McCulloch WS, Pitts WH (1959) What the frogs eye tells the frogs brain. P Ire 47:1940-1951. CrossRef

Linke R, Roth G, Rottluff B (1986) Comparative studies on the eye morphology of lungless salamanders, family plethodontidae, and the effect of miniaturization. J Morphol 189:131-143. CrossRef Medline

Martinez-Conde S, Macknik SL (2008) Fixational eye movements across vertebrates: comparative dynamics, physiology, and perception. J Vis 8:28 21-16. CrossRef Medline
Mehta B, Schaal S (2002) Forward models in visuomotor control. J Neurophysiol 88:942-953. Medline

Mischiati M, Lin HT, Herold P, Imler E, Olberg R, Leonardo A (2015) Internal models direct dragonfly interception steering. Nature 517:333-338. CrossRef Medline

Monroy JA, Nishikawa KC (2009) Prey location, biomechanical constraints, and motor program choice during prey capture in the tomato frog, Dyscophus guineti. J Comp Physiol 195:843-852. CrossRef Medline

Mronz M, Lehmann FO (2008) The free-flight response of Drosophila to motion of the visual environment. J Exp Biol 211:2026-2045. CrossRef Medline

Muijres FT, Elzinga MJ, Melis JM, Dickinson MH (2014) Flies evade looming targets by executing rapid visually directed banked turns. Science 344:172-177. CrossRef Medline

Nijhawan R (1994) Motion extrapolation in catching. Nature 370:256-257. CrossRef Medline

Nijhawan R (2002) Neural delays, visual motion and the flash-lag effect. Trends Cogn Sci 6:387. CrossRef Medline

Olberg RM, Worthington AH, Venator KR (2000) Prey pursuit and interception in dragonflies. J Comp Physiol 186:155-162. CrossRef Medline

Perry RJ, McNaughton PA (1991) Response properties of cones from the retina of the tiger salamander. J Physiol 433:561-587. CrossRef Medline

Roth G (1976) Experimental analysis of prey catching behavior of Hydromantes: Italicus Dunn (Amphibia, Plethodontidae). J Comp Physiol 109: 47-58. CrossRef

Roth G (1987) Visual behavior in salamanders. Berlin: Springer.

Rowell CHF, Pearson KG (1983) Ocellar input to the flight motor system of the locust: structure and function. J Exp Biol 103:265-288.

Tammero LF, Dickinson MH (2002) The influence of visual landscape on the free flight behavior of the fruit fly Drosophila melanogaster. J Exp Biol 205:327-343. Medline

von Reyn CR, Breads P, Peek MY, Zheng GZ, Williamson WR, Yee AL, Leonardo A, Card GM (2014) A spike-timing mechanism for action selection. Nat Neurosci 17:962-970. CrossRef Medline

Wolpert DM, Ghahramani Z, Jordan MI (1995) An internal model for sensorimotor integration. Science 269:1880-1882. CrossRef Medline

Zago M, McIntyre J, Senot P, Lacquaniti F (2009) Visuo-motor coordination and internal models for object interception. Exp Brain Res 192:571604. CrossRef Medline

Zangemeister WH, Jones A, Stark L (1981) Dynamics of head movement trajectories: main sequence relationship. Exp Neurol 71:76-91. CrossRef Medline 